\title{
Inhibition of enhanced toxin production by Clostridium difficile in biotin-limited conditions
}

\author{
K. YAMAKAWA, T. KARASAWA, T. OHTA*, H. HAYASHI† and S. NAKAMURA \\ Department of Bacteriology, School of Medicine, Kanazawa University, Kanazawa, Ishikawa 920-8640, \\ * College of Medical Technology and Nursing and †Department of Microbiology, Institute of Basic Medical \\ Sciences, University of Tsukuba, Tshukuba, Ibaraki 305, Japan
}

\begin{abstract}
Production of toxins $\mathrm{A}$ and $\mathrm{B}$ by Clostridium difficile is enhanced in a defined medium with biotin-limited conditions. In the present study compounds inhibitory to enhanced toxin production by a $C$. difficile strain were examined. Increases in biotin concentration from $0.05 \mathrm{nM}$ to $50 \mathrm{nM}$ accelerated growth and inhibited enhanced toxin production. Asparagine, glutamic acid and glutamine (10 mM) showed an effect on growth and toxin production similar to that of biotin. Lysine $(10 \mathrm{~mm})$ suppressed growth and inhibited toxin production. Addition of these toxin-inhibitory compounds within an incubation period of 2 days inhibited the enhanced toxin production, but later addition showed only slight inhibition of toxin production. Amino acids contained in the defined medium under the biotin-limited conditions were actively utilised in the presence of the three toxin-inhibitory amino acids, but in the presence of lysine, amino-acid utilisation was suppressed. Different mechanisms of action of these toxin-inhibitory molecules, which may be divided into excess biotin, asparagine-glutamic acid-glutamine group, and lysine, are discussed.
\end{abstract}

\section{Introduction}

Clostridium difficile is the pathogen responsible for most cases of pseudomembranous colitis and many cases of antibiotic-associated diarrhoea. The bacterium produces toxins $\mathrm{A}$ and $\mathrm{B}[1-5]$. An earlier study showed that the production of these toxins by $C$. difficile is enhanced in a biotin-limited defined medium (biotin effect) and it was hypothesised that administration of excess biotin to a patient with $C$. difficile colitis may have a therapeutic effect [6].

Enzymes containing biotin as a prosthetic group play significant roles in various physiological pathways, such as the metabolism of glucose, amino acids, fatty acids and nucleic acids [7]. As overall deterioration in cell physiology due to biotin insufficiency may be related to enhanced toxin production, it is difficult to determine the direct contribution of biotin to toxin regulation.

Nine amino acids - tryptophan, methionine, valine, isoleucine, proline, leucine, cysteine, glycine and threonine [8] - from the 18 amino acids in the defined medium of Haslam et al. [9] were selected for

Received 1 Oct. 1997; accepted 4 Jan. 1998.

Corresponding author: Professor S. Nakamura. the development of a defined medium to achieve better toxin production. The first seven amino acids are essential for bacterial growth and the last two are growth-enhancing $[6,10]$. Toxin production by $C$. difficile was higher in this defined medium than in Haslam's medium. Therefore, it was likely that an omitted amino acid(s) suppresses toxin production by C. difficile.

The present study examined the inhibitory effect of certain amino acids, as well as excess biotin, on enhanced toxin production by $C$. difficile in a biotinlimited defined medium and analysed changes in amino-acid consumption in media with and without inhibitory amino acids.

\section{Materials and methods}

Bacterial strain

A toxigenic $C$. difficile strain KZ 1647 was used. This strain showed a typical response of enhanced toxin production in a biotin-limited defined medium [6].

\section{Defined medium and cultivation}

A biotin-limited defined medium [6] containing biotin $(0.05 \mathrm{nM})$ and the following amino acids, tryptophan, 
methionine, valine, isoleucine, proline, leucine, cysteine, glycine and threonine, was used. The medium was inoculated for toxin production as described previously [6] and incubated anaerobically at $37^{\circ} \mathrm{C}$ for 5 days. To evaluate the effect of a test compound, $0.2 \mathrm{ml}$ of the concentrated solution $(\mathrm{pH} \mathrm{7.4)}$ was added to a culture medium $(10 \mathrm{ml})$ while flushing with oxygen-free gas. The same volume of saline was added to other tubes of culture medium as a negative control.

\section{Bacterial growth and toxin assay}

Bacterial growth was determined by measuring the optical density of cultures at $560 \mathrm{~nm}$ as described previously [6]. Levels of toxins A and B in culture supernates and in sonicated cell extracts were determined by an enzyme-linked immunosorbent assay and by a conventional cytotoxicity assay with baby hamster kidney cells, respectively, as reported previously [6]. Experiments were conducted in duplicate and mean values are presented.

\section{Amino acids analysis}

Amino acid concentrations in membrane-filtered (Millex GV, Millipore, Yonezawa, Japan) cultures were determined with a JLC-300 amino acid analyser (JEOL, Tokyo, Japan).

\section{Results}

\section{Inhibition of $C$. difficile toxin production with excess biotin}

Biotin ( $50 \mathrm{nM}$ ) was added to cultures of strain KZ 1647 at different incubation periods and then bacterial growth and toxin production were determined. Excess biotin increased bacterial growth within 1 day of its addition to the cultures (Fig. 1). Addition of biotin at early incubation times yielded a remarkable response in growth. Furthermore, a growth increase was still shown by addition to 4-day-old cultures. Toxin production was clearly inhibited by the addition of biotin to 1- and 2day-old cultures; toxin A levels did not exceed $200 \mathrm{ng} / \mathrm{ml}$, about one-tenth of the level $(1880 \mathrm{ng} / \mathrm{ml})$ achieved in biotin-limited conditions (Fig. 2). However, in the cultures to which biotin was added on the third day of incubation or later, the toxin level was similar to that produced in biotin-limited conditions. Toxin B levels paralleled those of toxin A.

\section{Inhibition of toxin production by amino acids}

Toxin production by strain KZ 1647 was examined in the biotin-limited defined medium supplemented with nine amino acids $(\mathrm{g} / \mathrm{L})$ : alanine $(0.2)$, arginine $(0.2)$, aspartic acid (0.3), glutamic acid (0.9), histidine (0.1), lysine $(0.3)$, phenylalanine $(0.2)$, serine $(0.3)$ and tyrosine $(0.1)$ (concentration of supplemented amino acid was the same as that in complete amino acid-
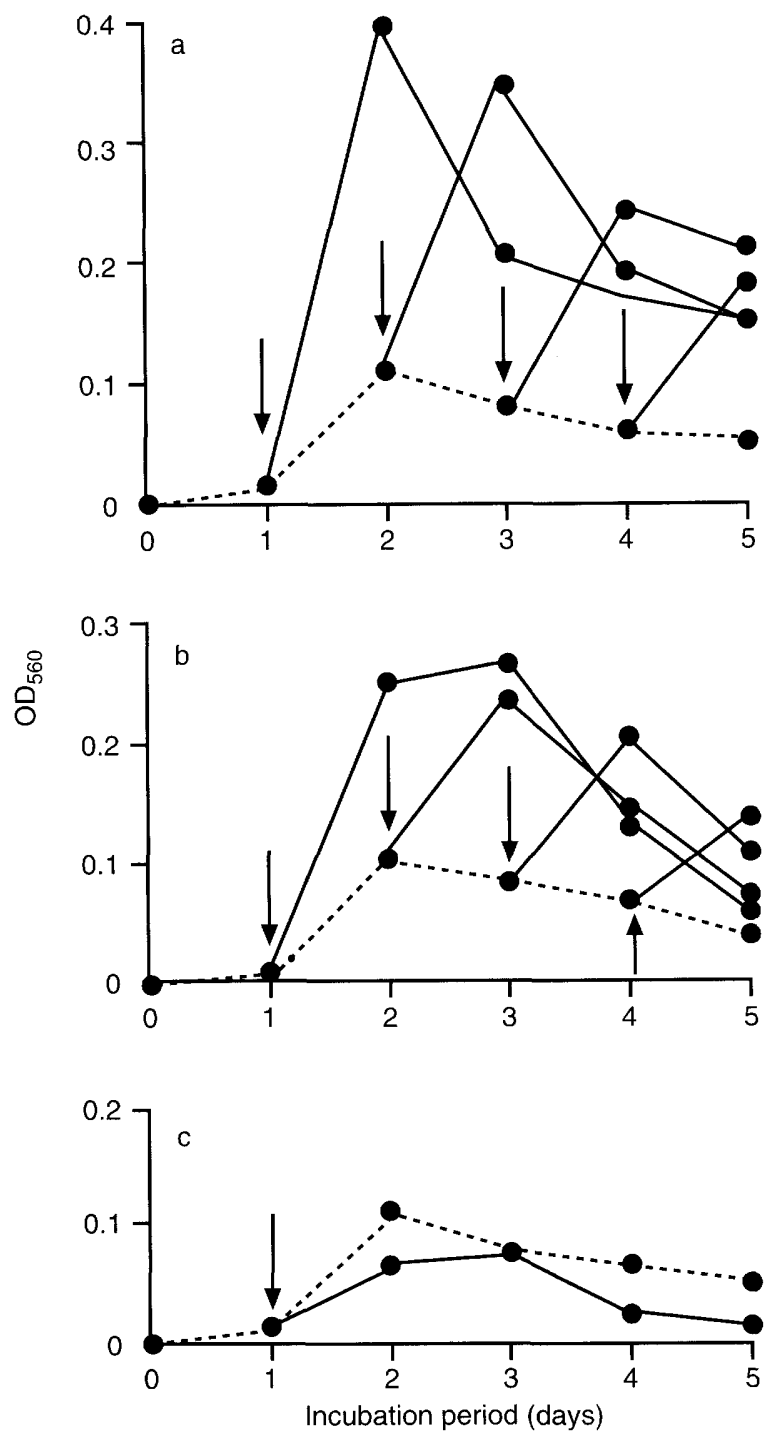

Fig. 1. Effect of biotin increase or addition of glutamine and lysine on growth of $C$. difficile $\mathrm{KZ} 1647$. a, $50 \mathrm{nM}$ biotin; b, $10 \mathrm{mM}$ glutamine; c, $10 \mathrm{mM}$ lysine. Timing of addition is indicated by arrows. Dotted base line is a growth curve in biotin-limited conditions.

defined medium [9]). Toxin levels were low compared to those in biotin-limited defined medium without the supplementation. The levels of toxins $\mathrm{A}$ and $\mathrm{B}$ were $2050 \mathrm{ng} / \mathrm{ml}$ and $2^{14.0} \mathrm{CU} / 50 \mu \mathrm{l}$ in the non-supplemented medium and $<10 \mathrm{ng} / \mathrm{ml}$ and $2^{3.5} \mathrm{CU} / 50 \mu \mathrm{l}$, respectively, in the supplemented medium.

On the basis of these findings, single amino acids used in the above supplementation experiment were added to the medium at $10 \mathrm{~mm}$, and toxin production was determined to identify amino acid(s) inhibitory to the enhanced toxin production. An inhibitory effect was observed with lysine, asparagine, glutamic acid and glutamine (Table 1); the levels of toxins A and B were $\leqslant 85 \mathrm{ng} / \mathrm{ml}$ and $\leqslant 2^{3.0} \mathrm{CU} / 50 \mu \mathrm{l}$, respectively. Intracellular toxin titres in the cultures with inhibitory amino acids were low; levels of toxins $\mathrm{A}$ and $\mathrm{B}$ were $<10 \mathrm{ng} / \mathrm{ml}$ and $2 \mathrm{CU} / 50 \mu \mathrm{l}$, respectively, in the cultures with asparagine, glutamic acid or glutamine, 

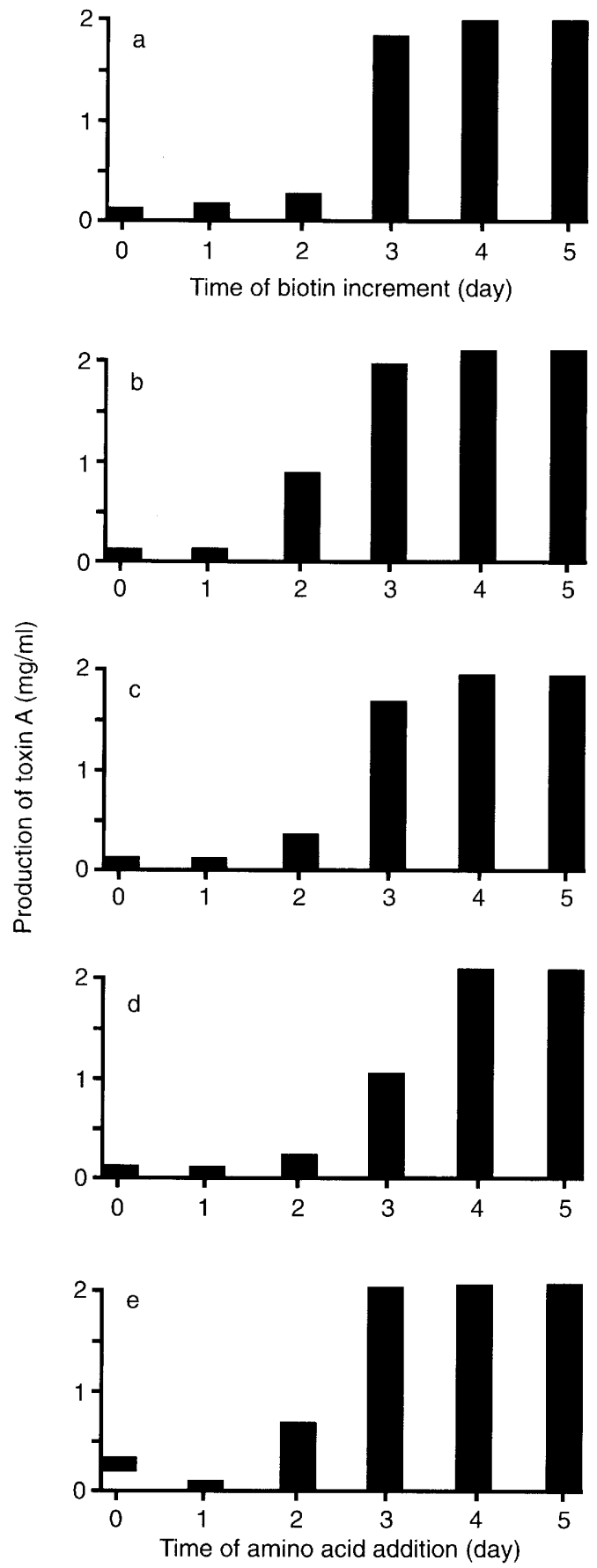

Fig. 2. Inhibition of enhanced toxin A production by $C$. difficile $\mathrm{KZ} 1647$ by biotin increase or addition of inhibitory amino acids. a, $50 \mathrm{nM}$ biotin; b, $10 \mathrm{~mm}$ asparagine; c, $10 \mathrm{~mm}$ glutamic acid; d, $10 \mathrm{mM}$ glutamine; e, $10 \mathrm{~mm}$ lysine. Toxin A titre was determined after incubation for 5 days.

and $12 \mathrm{ng} / \mathrm{ml}$ and $2 \mathrm{CU} / 50 \mu \mathrm{l}$, respectively, in the cultures with lysine. Intracellular toxin A and B levels in the cultures without the inhibitory amino acids were $70 \mathrm{ng} / \mathrm{ml}$ and $2^{8} \mathrm{CU} / 50 \mu \mathrm{l}$, respectively.

To analyse further the inhibitory effect of the four amino acids, they were added after different periods of incubation and then growth and toxin production were determined. When glutamine was added to 1-day-old cultures, bacterial growth increased by about double
Table 1. Inhibitory effect of amino acids on toxin production by $C$. difficile $\mathrm{KZ} 1647$ in a biotin-limited defined medium

\begin{tabular}{lcc}
\hline $\begin{array}{l}\text { Amino acid } \\
\text { added }(10 \mathrm{~mm})^{*}\end{array}$ & $\begin{array}{c}\text { Toxin A } \\
(\mathrm{ng} / \mathrm{ml})\end{array}$ & $\begin{array}{c}\text { Toxin B } \\
\left(\log _{2} \mathrm{CU} / 50 \mu \mathrm{l}\right)\end{array}$ \\
\hline None & 1940 & 14.5 \\
Aspartic acid & 1810 & 14.5 \\
Alanine & 1270 & 14.0 \\
Phenylalanine & 1220 & 14.0 \\
Serine & 1070 & 14.0 \\
Arginine & 1010 & 13.5 \\
Histidine & 730 & 12.5 \\
Lysine & 85 & 5.0 \\
Asparagine & 38 & 5.0 \\
Glutamic acid & $<10$ & 3.0 \\
Glutamine & $<10$ & 3.5 \\
\hline
\end{tabular}

${ }^{*}$ The basal defined medium was biotin-limited $(0.05 \mathrm{nM})$ and contained nine amino acids: tryptophan, methionine, valine, isoleucine, proline, leucine, cysteine, glycine and threonine.

the maximum growth of cultures without glutamine (Fig. 1). A growth increase still occurred with the addition of glutamine to 2-4-day-old cultures. The addition of glutamic acid or asparagine showed a similar effect on growth that was not as marked as with glutamine (data not shown). However, with lysine, growth was suppressed following addition to 1-day-old cultures and lysine added after incubation for 2 days or later showed no influence on growth (data not shown). The addition of glutamine to 1- or 2-day-old cultures almost completely inhibited toxin A production, while for 3-day cultures glutamine inhibited toxin A to about half the level produced in the cultures without glutamine (Fig. 2). The addition of asparagine, glutamic acid, or lysine to 1- or 2-day-old cultures was also highly inhibitory and toxin B levels in these cultures paralleled those of toxin A.

Amino-acid consumption in the presence of the inhibitory amino acid

Amino-acid concentrations in culture supernates after incubation of strain KZ 1647 for 5 days with and without inhibitory amino acids were determined. The inhibitory amino acids were added at the beginning of incubation in this experiment. In cultures without inhibitory amino acids, threonine was almost totally consumed, as was approximately half of the leucine, isoleucine and proline (Table 2). Valine, methionine, tryptophan and glycine were not significantly utilised. When the inhibitory amino acid was added to the medium, asparagine, glutamic acid, glutamine and lysine were consumed in amounts of $1.14,1.79,4.75$ and $1.53 \mu \mathrm{mol} / \mathrm{ml}$, respectively. Differences in leucine and isoleucine consumption were observed in media containing inhibitory amino acids. Consumption of leucine and isoleucine was increased in media containing asparagine, glutamic acid or glutamine, but consumption in the medium containing lysine was similar to that in the medium without inhibitory amino acids. This feature was observed also for valine and methionine. 
Table 2. Concentration of amino acids in the biotin-limited medium after incubation of strain KZ 1647 for 5 days

\begin{tabular}{|c|c|c|c|c|c|c|c|c|}
\hline \multirow[b]{2}{*}{ Amino acid added } & \multicolumn{8}{|c|}{ Amino-acid concentration ( $\mathrm{mM})$} \\
\hline & Thr & Leu & Ile & Pro & Val & Met & Trp & Gly \\
\hline None (uninoculated) & 3.36 & 18.30 & 13.72 & 15.63 & 15.36 & 8.04 & 2.94 & 2.66 \\
\hline None & 0.34 & 8.85 & 6.21 & 9.10 & 15.02 & 8.13 & 2.84 & 2.65 \\
\hline $10 \mathrm{~mm}$ Asn & 0.40 & 0.00 & 4.48 & 8.20 & 13.44 & 6.74 & 2.67 & 2.59 \\
\hline $10 \mathrm{~mm}$ Glu & 0.03 & 2.27 & 4.80 & 9.62 & 13.96 & 6.66 & 2.75 & 2.49 \\
\hline $10 \mathrm{~mm}$ Gln & 0.01 & 2.01 & 4.25 & 8.49 & 13.27 & 6.80 & 2.69 & 2.41 \\
\hline $10 \mathrm{mM}$ Lys & 0.52 & 8.79 & 6.65 & 8.11 & 15.07 & 8.06 & 2.86 & 2.62 \\
\hline
\end{tabular}

Mean concentrations from triplicate cultures in each medium are presented. The cysteine concentration has been omitted because an accurate analysis was not performed.

\section{Discussion}

As well as excess biotin, four amino acids were shown to be inhibitory to enhanced toxin production by $C$. difficile in biotin-limited conditions. Growth of $C$. difficile KZ 1647 recovered following biotin supplementation. This growth recovery may reflect 'normal' physiology of the bacterial cells, which is related to a certain relaxed state of toxin production. The addition of excess biotin to cultures after incubation for up to 2days, the early phase of toxin production [6], caused such relaxation. However, biotin added from the third day of incubation did not inhibit enhanced toxin production, even though a considerable increase in vegetative growth was observed. These findings suggest that enhanced toxin production includes an irreversible course of cell physiology. Toxin synthesis by $C$. difficile in a complex medium may be related to early events of sporulation [11]. In the present system, the decline phase of growth of strain KZ 1647 started after incubation for c. 2 days and sporulation then occurred (unpublished data). Therefore, it is likely that the irreversible process of enhanced toxin production is linked to a physiological change from vegetative growth to sporulation.

Amino acids regulate neurotoxin production by $C$. botulinum in defined media: excess arginine and tryptophan markedly decrease neurotoxin production by $C$. botulinum type $\mathrm{A}$ and $\mathrm{B}$ strains, and type $\mathrm{E}$ strains, respectively $[12,13]$. In the latter case, tryptophan is an essential nitrogen source in a minimal medium. Leyer and Johnson [13] suggested that decreased toxin titres were related specifically to tryptophan metabolism, although growth was not affected by excess tryptophan. They also suggested that toxin repression by tryptophan may indicate nutritional sufficiency of the cell and pointed out the importance of other nitrogen sources in the control of toxin production. Both reports $[12,13]$ further described a similar effect with glutamic acid to some degree, although glutamic acid did not increase growth.

The defined medium used in the present study contained the minimal number of amino acids for good growth of $C$. difficile [9]. The amino acids inhibitory to toxin production, i.e., asparagine, gluta- mic acid, glutamine and lysine, have no affect on the growth of $C$. difficile $[9,10]$. However, under biotinlimited conditions, glutamine, glutamic acid and asparagine yielded better growth than the minimal combination of amino acids. Toxin inhibition by glutamic acid was similar to that seen with neurotoxin production by $C$. botulinum, although $C$. difficile growth was increased to some degree. Glutamine, glutamic acid and asparagine play central roles in clostridial physiology such as in amino-acid catabolism [14]. Therefore, availability of these three amino acids may compensate a biased physiology with low functional biotin enzymes.

Lysine biosynthesis is achieved through many enzymic steps in the genus Clostridium as with other genera [14]. However, lysine is not essential for growth of $C$. difficile $[9,10]$, and is growth suppressive under biotin-limited conditions. The inhibition of toxin production by lysine may be different to that caused by asparagine, glutamic acid and glutamine mentioned above.

The molecular mechanisms of $C$. difficile toxin regulation have been studied progressively. Recently, it was shown that transcriptional activation of the toxin A and B genes is induced at the postexponential growth phase in a complex medium and it was suggested that positive and negative regulation genes control the expression of these toxin genes [15-18]. It will be interesting to learn whether intracellular biotin concentration influences the function or expression of regulation proteins. In any case, knowledge of the physiological background of $C$. difficile cells is necessary for any investigation of $C$. difficile toxigenesis at the molecular level.

The nutritional environment in the human colon (the site of $C$. difficile infection) is different to our simplified model due to the presence of non-digested polysaccharides and mucopolysaccharides with trace amounts of glucose, and an unknown balance of amino acids and polypeptides. Moreover, the intestinal environment is dramatically changed both bacteriologically and nutritionally when antibiotics are administered. For these reasons the mechanism of C. difficile toxin production should be further elucidated. 
We are grateful to the Chemical Analysis Centre, University of Tsukuba, for amino acid analysis.

\section{References}

1. Lyerly DM, Krivan HC, Wilkins TD. Clostridium difficile: its disease and toxins. Clin Microbiol Rev 1988; 1: 1-18.

2. Dove $\mathrm{CH}$, Wang $\mathrm{S}-\mathrm{Z}$, Price SB et al. Molecular characterization of the Clostridium difficile toxin A gene. Infect Immun 1990; 58: 480-488.

3. Johnson JL, Phelps C, Barroso L, Roberts MD, Lyerly DM, Wilkins TD. Cloning and expression of the toxin B gene of Clostridium difficile. Curr Microbiol 1990; 20: 397-401.

4. Just I, Wilm M, Selzer J et al. The enterotoxin from Clostridium difficile (ToxA) monoglucosylates the Rho proteins. J Biol Chem 1995; 270: 13932-13936.

5. Just I, Selzer J, Wilm M, von Eichel-Streiber C, Mann M, Aktories $\mathrm{K}$. Glucosylation of Rho proteins by Clostridium difficile toxin B. Nature 1995; 375: 500-503.

6. Yamakawa K, Karasawa T, Ikoma S, Nakamura S. Enhancement of Clostridium difficile toxin production in biotin-limited conditions. J Med Microbiol 1996; 44: 111-114.

7. Lehninger AL, Nelson DL, Cox MM. Principles of biochemistry, 2nd edn. New York, Worth Publishers. 1993.

8. Yamakawa K, Kamiya S, Meng XQ, Karasawa T, Nakamura S. Toxin production by Clostridium difficile in a defined medium with limited amino acids. J Med Microbiol 1994; 41: 319-323.

9. Haslam SC, Ketley JM, Mitchell TJ, Stephen J, Burdon DW Candy DCA. Growth of Clostridium difficile and production of toxins $\mathrm{A}$ and $\mathrm{B}$ in complex and defined media. $\mathrm{J} \mathrm{Med}$
Microbiol 1986; 21: 293-297.

10. Karasawa T, Ikoma S, Yamakawa K, Nakamura S. A defined growth medium for Clostridium difficile. Microbiology 1995; 141: $371-375$.

11. Kamiya S, Ogura $H$, Meng $X Q$, Nakamura S. Correlation between cytotoxin production and sporulation in Clostridium difficile. J Med Microbiol 1992; 37: 206-210.

12. Patterson-Curtis SI, Johnson EA. Regulation of neurotoxin and protease formation in Clostridium botulinum Okra B and Hall A by arginine. Appl Environ Microbiol 1989; 55: 1544-1548.

13. Leyer GJ, Johnson EA. Repression of toxin production by tryptophan in Clostridium botulinum type E. Arch Microbiol 1990; 154: 443-447.

14. Andreesen JR, Bahl H, Gottschalk G. Introduction to the physiology and biochemistry of the genus Clostridium. In: Minton NP, Clarke DJ (eds) Clostridia. New York, Plenum Press. 1989: 27-62.

15. Braun V, Hundsberger T, Leukel P, Souerborn M, von EichelStreiber C. Definition of the single integration site of the pathogenicity locus in Clostridium difficile. Gene 1996; 181: $29-38$.

16. Hammond GA, Lyerly DM, Johnson JL. Transcriptional analysis of the toxigenic element of Clostridium difficile. Microb Pathog 1997; 22: 143-154.

17. Hundsberger T, Braun V, Weidmann M, Leukel P, Sauerborn M von Eichel-Streiber $C$. Transcription analysis of the genes $t c d A-E$ of the pathogenicity locus of Clostridium difficile. Eur $J$ Biochem 1997; 244: 735-742.

18. Moncrief JS, Barroso LA, Wilkins TD. Positive regulation of Clostridium difficile toxins. Infect Immun 1997; 65: 1105-1108. 